\title{
Modus in rebus
}

\author{
Maria Linda Falcidieno \\ Enrica Bistagnino \\ Alessandro Castellano \\ Massimo Malagugini \\ Ruggero Torti \\ Maria Elisabetta Ruggiero
}

\section{Abstract}

Il tema affrontato in questa sede è strettamente connesso alle recenti vicende legate alla pandemia e ai periodi di clausura totale; abbiamo imparato a convivere con noi stessi o con una strettissima cerchia famigliare e abbiamo imparato ad utilizzare l'informatica come un supporto "amichevole" e non come un mero programma da imparare. Ci siamo resi conto delle distanze fisiche, che credevamo di aver per sempre annullato con la capacità di viaggiare in tempi sempre più contenuti. Abbiamo toccato con mano la solitudine e il senso di marginalità dell'esistenza.

$\mathrm{Ma}$ - riferendosi all'esperienza universitaria didattica e di ricerca - tutto questo ha anche contribuito in modo sorprendente a potenziare il nostro modo di lavorare: il nostro ruolo culturale e operativo ne è uscito senza dubbio arricchito, a tal punto da travalicare il corretto equilibrio tra rapporto "in presenza" e "a distanza", fino a confondere i piani, come se le sole distanze fisiche fossero l'unico impedimento per una comunicazione effettiva.

In queste brevi note si vuole, invece, porre in evidenza come esistano altre "distanze", non fisiche, ma forse ancor più limitanti per la comprensione reciproca e come un'occasione didattica sia stata l'inizio per riflessioni teorico-metodologiche e approccio alla ricerca su questo tema.

Parole chiave

segni, grafie, cultura, appartenenza.

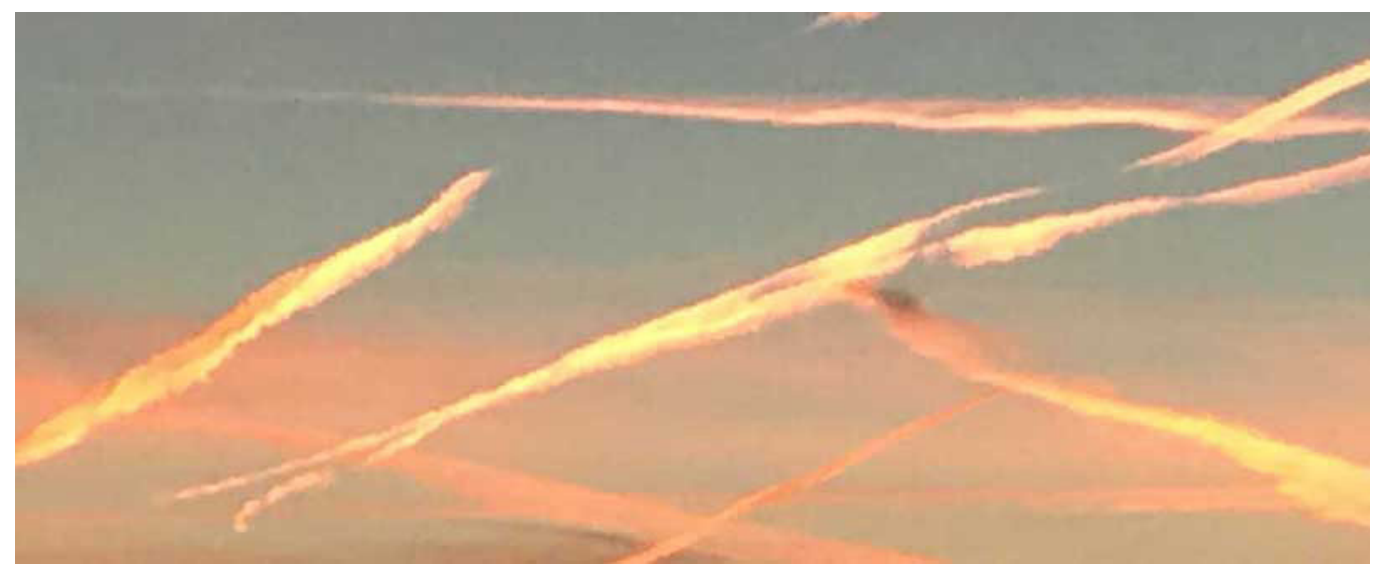




\section{Introduzione}

C'è un tempo per ogni cosa e c'è una modalità (di sviluppo, di percezione, di appropriazione...) per ogni cosa. II tema della distanza comunicativa legata alle tecnologie che permettano di superarla e ai linguaggi possibili che derivano dall'utilizzo di dette tecnologie appare come una irrinunciabile opportunità per riflettere e per comprendere punti di vista forse inusuali, ma certo utili per una corretta comprensione del problema (e/o dell'opportunità) che l'attuale momento pandemico ha messo in chiara evidenza $[\mathrm{l}]$.

In questa sede, tuttavia, si vuole trattare un aspetto peculiare della comunicazione, che vede la distanza non solo come distanza 'fisica', bensì anche come distanza 'percettivo-culturale', in parte risolvibile con strumenti, tecniche e metodologie di approccio, ma in parte eliminabile (o, quantomeno, ridimensionabile) solo con un cambio di passo e con la consapevolezza che la globalità sta nella condivisione e non solo nella velocità e nella potenza dei mezzi oggi a disposizione.

L'occasione: la docenza delle discipline della grafica presso una università cinese [2]; il tema: il linguaggio (verbale e non verbale); l'ottica di indagine: il rapporto che lega le nuove tecnologie alla possibilità di accorciare le distanze tra un patrimonio culturale eminentemente visivo (quello dell'espressività dei popoli orientali) e uno suddiviso in espressioni alfabetiche e di immagine. Da qui, il toccare con mano che non sempre la sola tecnologia avvicina, ma che sempre ciò accade quando si condividono alcuni fondamenti necessari alla comprensione reciproca e come, quindi, occorra consapevolezza dei limiti (oltre che delle potenzialità) delle tecnologie contemporanee per supportarle e accompagnarle.

Perché dall'esperienza tutt'ora in corso è emerso con chiarezza come sia fondamentale il condividere in profondità e non solo a livello nozionistico il patrimonio di espressioni 'di base' - 'codici', come ben sappiamo noi ricercatori nell'ambito della disciplina della rappresentazione - , che sono la condizione necessaria (se pure non sufficiente) perché la comunicazione tra emittenti e riceventi funzioni senza possibilità di fraintendimenti, ambiguità, errori; soprattutto in ambito di formazione, avere una condivisione o quantomeno una profonda conoscenza dei reciproci codici espressivi significa evitare quella sorta di prevaricazione che si attua quando chi fornisce le informazioni e guida le espressioni visive progettuali illustra e tende a rendere uniformi le finalità progettuali, rendendole aderenti con il proprio bagaglio culturale [3].

Nel progetto visivo, si possono riscontrare rilevanti difficoltà nella messa a sistema di testi e immagini, nel loro proporzionamento, nell'organizzazione delle gerarchie e degli spazi, nella scelta dei caratteri tipografici maggiormente adeguati al target di riferimento e al fine della comunicazione, se non si conoscono e si comprendono le ragioni profonde delle scelte, se non si condivide una cornice di riferimenti culturali; in questo caso, la comprensione linguistica non è affatto sufficiente a garantire una buona resa del prodotto: testi in inglese permettono di comprendere i significati letterali del messaggio, ma tutta la visualità risulta pericolosamente in bilico tra i dettami di chi in quel momento trasferisce competenze e chi li attua, cercando di adeguare la propria storia a una estremamente lontana e poco nota [4]. La tecnologia, in questo caso, aiuta ed è necessaria per lo svolgimento della formazione a distanza, ma nulla può fare per colmare l'altra distanza, quella più profonda e strutturale; in maniera analoga, gli strumenti informatici realizzati con codici linguistici diversi per matrice (in sostanza e per una brutale definizione di massima: pittografica o alfabetica) rendono estremamente difficoltoso l'operare in contemporanea - docente e discente - fino a impedire a volte la creazione di quell'empatia e quella spontaneità di rapporto che è la caratteristica primaria per la buona riuscita di una attività laboratoriale.

Quali le considerazioni che sono derivate dalle riflessioni fatte?

Innanzitutto il riconoscere l'importanza e la necessità delle tecnologie informatiche e del conseguente ruolo assunto nella formazione, che ormai nessuno più nega; ma subito dopo il riconoscere che occorre una unità di intenti, che travalica la nazionalità e la singola applicazione della conoscenza, per rendere ineludibile uno spostamento di visuale: occorre operare nella ricerca di una sorta di 'esperanto' [5] della visualità, che stabilisca alcuni prin- 
cipi che possano garantire la riuscita dei progetti visivi a prescindere dalla loro collocazione geo-culturale. Operare per una globalità intesa come condivisione di codici e conseguente comprensione.

D'altra parte, questo è ciò che il momento richiede in ogni ambito e soprattutto nella condivisione del rispetto del mondo che ci ospita e che ci sta lanciando segni sempre più chiari di cedimento [6].

Allora, forse, non si tratta solo di Modus in rebus, bensì dell'antico monito sul rispetto dei limiti e della ricerca di equilibrio:"est modus in rebus. sunt certi denique fines, quos ultra citraque nequit consistere rectum" (Orazio, Satire ed epistole) [La Penna 1967, pp. I6].

Di seguito, si propongono cinque focus di ricerca: Riflettere, Percepire, Diversificare, Condividere, Riferire. (ML.F.).

\section{Riflettere (Product Image Design Laboratory)}

Fig. I Product Image Design Laboratory, 2020/2021

prof.ssa Enrica Bistagnino.

Elaborato di uno

studente sul tema

dell anticonformismo.

Beijing University of

Chemical Technology,

BUCT.

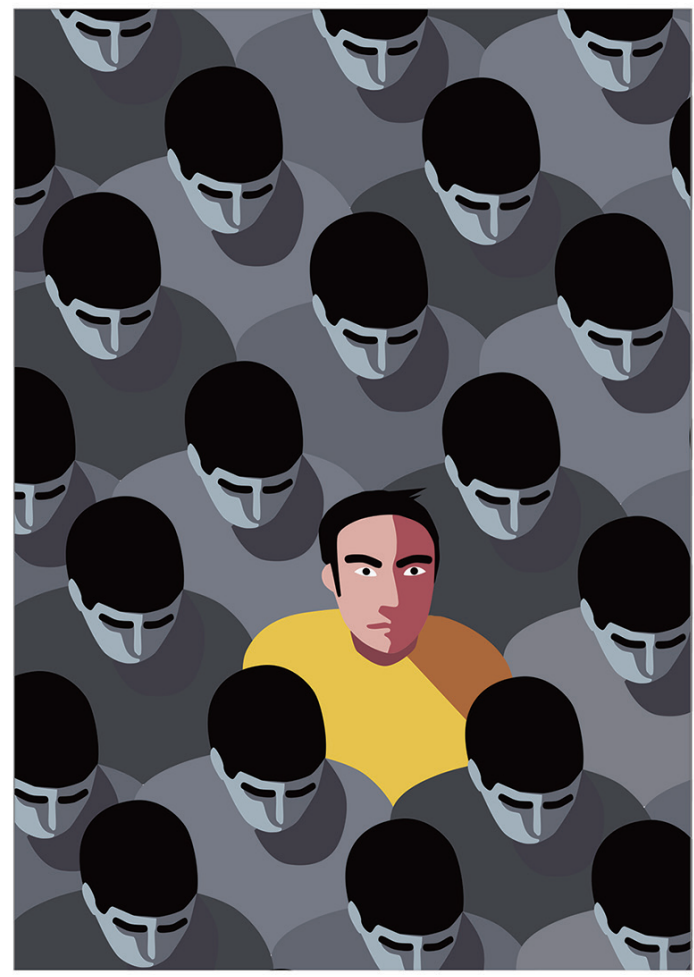

La potenza dialogica dell'immagine è oggi più che mai evidente e fondamentale.

Ogni relazione è mediata da rappresentazioni di luoghi, persone e segni che, prevalentemente in forma dinamica, appaiono sulle molteplici interfacce delle piattaforme web e nei dispositivi di visualizzazione.

Per quanto riguarda l'ambito della didattica, specificamente quella relativa alle tematiche della rappresentazione e comunicazione, nelle sperimentazioni effettuate si rileva un'interessante sovrapposizione di immagini: l'oggetto della riflessione, un artefatto visivo, è infatti mediato da ulteriori rappresentazioni - di spazi di contesto, di aule, di studenti - che aprono certamente molti temi di riflessione.

Nella consapevolezza della complessità dell'argomento si vuole, in questa sede, solo accennare ad alcuni temi emersi nell'esperienza didattica svolta presso la BUCT nel Product Image Design laboratory che, se da un lato ha ulteriormente affermato la portata comunicativa della rappresentazione, dall'altro ha anche sottolineato, come era prevedibile, quanto sia importante, per una conoscenza profonda di immagini 'distanti' dal nostro linguaggio visivo, accedere in presenza al contesto culturale che le ha generate. 
Infatti, l'eccezionale prossimità resa possibile dalle piattaforme per la didattica da remoto, sembra risentire, tuttavia, di una generale mancanza di profondità e di ampiezza di visione. Nella piattezza e nella delimitazione dello schermo, le diversità di codici e linguaggi che emergono nelle immagini, risultano, in una certa misura, porzioni residuali di una cultura che rimane lontana, ancora non pienamente accessibile.

Manca, nell'osservare queste immagini che 'si aprono' nelle interfacce dei dispositivi, il rinforzo generato dal contesto che costituisce una preziosa sorta di eco di estetiche e culture; manca il paesaggio, scenario denso di indizi culturali; manca la libertà dello sguardo di discretizzare segni orientando selettivamente la propria attenzione.

In altri termini, la visualità, demandata all'audio-visione, pur confermando il carattere 'cosmopolita' proprio del linguaggio visivo (carattere importantissimo della dimensione dell'immagine) necessita di quel fondamentale potenziamento visivo dato dall'attivazione multisensoriale ancora fortemente legata a processi immersivi 'reali' piuttosto che da connessioni virtuali.

Quindi, se da un lato lo schermo dei dispositivi digitali ha consentito, in questo periodo di pandemia, un collegamento sostanzialmente continuo fra gli individui del pianeta - sotto il profilo personale, sociale e lavorativo, formativo - se ha reso possibile creare un senso di comunità, rappresentando, attraverso le persone, una sorta di spazio 'metafisico', agito virtualmente, dall'altro ha svelato i limiti dell'interfaccia nella formazione a distanza che, inquadrata nello schermo, rileva ed esprime diversità ancora da comprendere e armonizzare appieno in un orizzonte culturale irriducibile alla mediazione degli strumenti di comunicazione a distanza.

Gli studenti della BUCT sono stati invitati a selezionare alcuni progetti grafici contemporanei, di cultura orientale, e ad analizzarne la struttura e i codici. I risultati sono stati interessanti, le immagini decisamente 'potenti', ma quale forza e chiarezza in più avrebbero avuto se le avessimo osservate e contestualizzate in uno scambio di sguardi in uno spazio del campus? (E.B.).

\section{Percepire (Product Image Design Laboratory)}
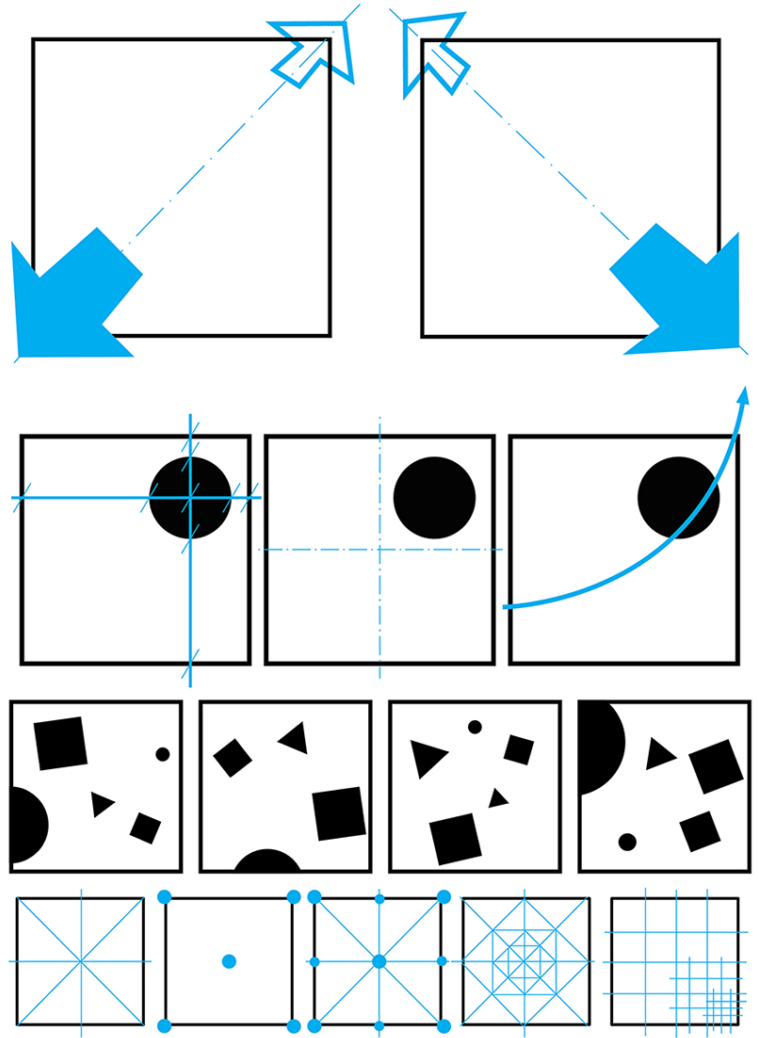
Fare lezione di percezione visiva in Cina e a distanza si è posta fin dall'inizio come una sfida. Normalmente i fenomeni percettivi vengono spiegati in base ad alcuni concetti legati all'esperienza della vita quotidiana. Ad esempio, per spiegare le forze attive in un campo non c'è niente di più utile che fare riferimento al nostro sistema di lettura: l'andamento da sinistra a destra e quello d'alto verso il basso indicano le direzioni e i versi delle due forze che fanno sì che l'angolo in basso a destra di una pagina rappresenti la parte più 'pesante' del campo. Ma come affrontare il tema con un modello di scrittura così diverso dal nostro? Nel cinese tradizionale la scrittura e la lettura procedono dall'alto in basso e da destra verso sinistra; il cinese contemporaneo invece dimostra una maggiore flessibilità e ha adottato la scrittura da sinistra a destra e, in molte occasioni, anche l'andamento orizzontale della scrittura, probabilmente molto per adattarsi al linguaggio del world wide web che ha imposto un modello organizzativo della pagina più occidentale. Ma se da una parte l'avvicinarsi del cinese contemporaneo al modello di scrittura occidentale potrebbe vedersi come un elemento facilitatore sulla didattica della percezione con la creazione di un contesto visivo condiviso, dall'altra rimane il dubbio di come e quanto il modello radicato della scrittura classica possa influire sul modo stesso di percepire la realtà.

Nel corso delle lezioni gli studenti cinesi hanno dimostrato una certa abilità ad apprendere e utilizzare i fenomeni della percezione legati alle forze generate dal campo e degli oggetti in esso contenuto. Con una certa facilità hanno capito i concetti legati alle diagonali armoniche o disarmoniche all'effetto ascesa o caduta rovinosa di composizioni costruite su quegli stessi elementi del campo. Ma non solo hanno dimostrato di capire il concetto in base alla lettura di immagini, sono stati anche in grado di fare loro il concetto negli elaborati realizzati durante il corso.

Con la stessa facilità gli studenti hanno affrontato gli esercizi di esplorazioni visiva dell'immagine sulla base degli esempi legati alla ricerca sui movimenti oculari condotta da Alfred L. Yarbus, riuscendo a cogliere i dettagli necessari per rispondere ai vari compiti visivi assegnati. Diverso il discorso per quanto riguarda il tema delle gerarchie visive. Se da un punto di vista teorico i concetti sono stati appresi senza problemi, nella pratica gli studenti cinesi hanno dimostrato una minore capacità organizzativa degli elementi in funzione dell'importanza delle singole parti.

Quanto osservato a lezione dà credito alla teoria che lega il nostro modo di percepire, e di progettare, intrinsecamente legato allo stile cognitivo dominante con il quale apprendiamo durante la nostra formazione. Lo stile cognitivo è fortemente influenzato dal contesto socioculturale in cui siamo cresciuti e, diversi studi, riportano come due estremi il modello olistico o globale tipico delle culture che vedono l'ordine naturale nella relazione tra le cose, come nella maggior parte delle culture orientali, e il modello analitico, basato su quello platonico, che lo vede nella categorizzazione e nell'analisi dei singoli oggetti. Da qui la facilità degli studenti cinesi ad apprendere fenomeni relazionali, funzionali e fortemente legati al contesto e la minore capacità di organizzazione discrezionale dei singoli elementi. (A.C.).

\section{Diversificare (Product Image Design Laboratory)}

Il linguaggio è la capacità peculiare degli esseri umani, di comunicare pensieri, esprimere sentimenti, e in genere di informare altri esseri sulla propria realtà interiore o sulla realtà esterna, per mezzo di un sistema di segni vocali o grafici; in senso ancora più ampio può intendersi come la facoltà di esprimersi attraverso altri segni, come gesti, simboli. La parola linguaggio chiama in causa anche il termine lingua, inteso come il complesso delle parole e locuzioni che un popolo utilizza per esprimere, comunicare, scambiare pensieri o sentimenti. Lingua a linguaggio si intrecciano inevitabilmente e, se da una parte conducono alla piena comprensione fra gli individui, dall'altra, possono portare a una 'Babele' di incomprensioni e differenze.

Questo è il rischio al quale si va incontro quando ci si trova ad affrontare il tema della comunicazione visiva - e dunque del codice visivo - con soggetti che provengono da paesi e culture particolarmente distanti dalla nostra, come è accaduto nell'ambito del Product Image 
Fig. 3. Dopo le sperimentazioni che hanno messo a confronto codici fonetici e codici ideografic nel tentativo di visualizzare il significato della parola stessa, si sono provati ad applicare i grafemi della scrittura ideografica per scrittura ideografica per fontico che sia in fonetico che sia in grado vocar la dal punto di vista visivo. Si e cosi introdotto termine di 'alfagramma' (elaborati degli studenti Hong Liu e Chen Boyu)
$B B B$

PROCESSO DI PROGETTAZIONE

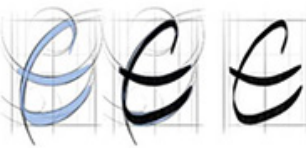

PROCESSO DI PROGETTAZIONE
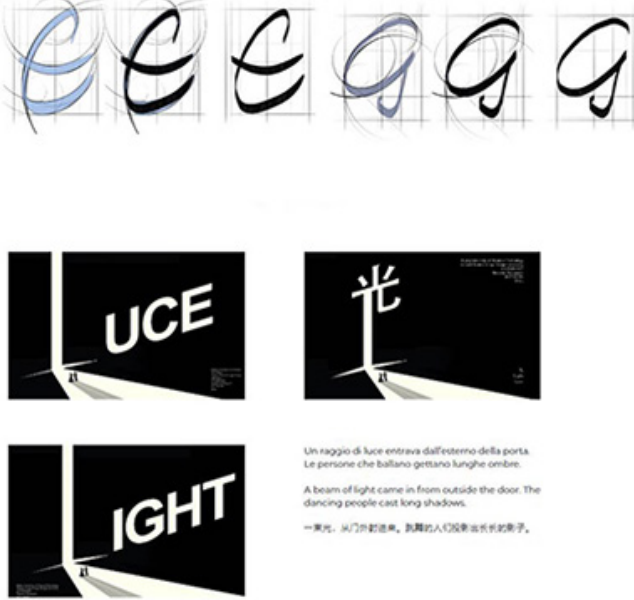

the

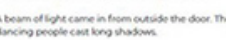

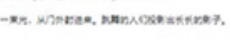

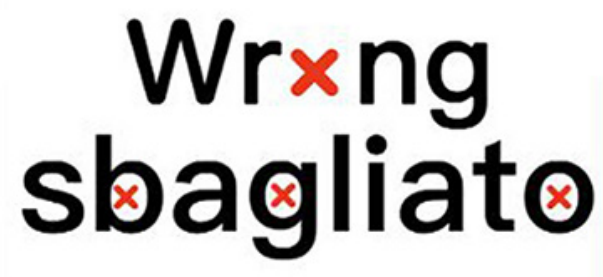

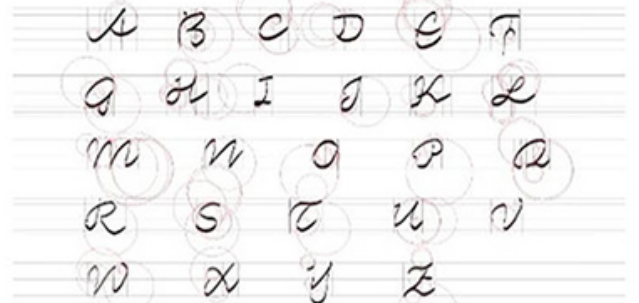

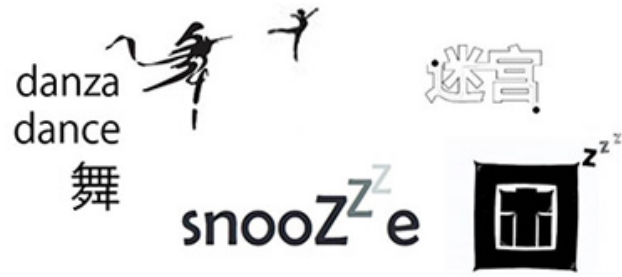

pencil

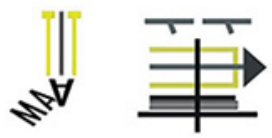

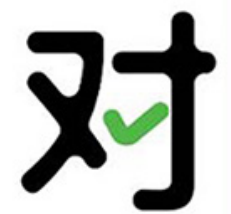

Design Laboratory. Le grandi differenze, che già emergono fino dallo studio dei più semplici codici visivi legati al colore, diventano ancora più evidenti nel momento in cui si analizza la parola, ovvero quella componente testuale che è parte integrante della comunicazione [Falcidieno 2006], in quanto, al di là dei diversi significati che ogni lingua attribuisce a parole equivalenti, (oviamente tradotte) si aggiunge una diversa logica nella rappresentazione della parola stessa. Ciò nasce dal fatto che ci si trova a porre a confronto una cultura che fonda la propria scrittura su un sistema ideografico, con la nostra che si basa su un sistema fonetico. Non si tratta, allora, soltanto di una diversa scrittura e/o pronuncia di uno stesso termine, bensì di una rappresentazione completamente differente che, inevitabilmente porta a percezioni visive altrettanto differenti.

In quest'ottica è stata esemplificativa l'analisi di progetti grafici che hanno elaborato la componente testuale fino a mettere in evidenza da un punto di vista visivo il significato della parola stessa. Questa operazione è risultata particolarmente interessante perché applicata non soltanto a una scrittura di tipo fonetico (come l'italiano o l'inglese), ma anche a una scrittura di tipo ideografico come quella cinese. II lavoro ha messo a fuoco differenti approcci agli aspetti visivi della parola scritta e ha mostrato sensibilità contrastanti nel recepire differenti accezioni stilistiche nell'elaborazione di un prodotto visivo. Se per la nostra cultura è scontato associare significati visivi diversi a una diversa tipologia di font, gamma seriale, maiuscole o minuscole, non altrettanto accade nella cultura orientale.

Anche le sperimentazioni opposte, ovvero quelle che provano a tradurre graficamente un alfabeto fonetico secondo grafemi ispirati alla scrittura ideografica, hanno portato a interessanti sperimentazioni che tentano di trovare connessioni quasi impossibili fra codici così differenti. (M.M.). 


\section{Condividere (Digital Representation e Product Image Design Laboratory)}

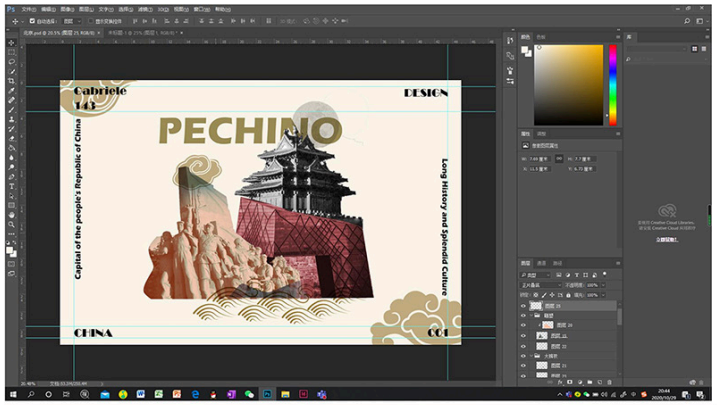

Fig. 4. Confronto tra interfaccia utente de software Adobe Photoshop in lingua cinese e in lingua italiana utilizzato dagli studenti cinesi per lo svolgimento delle ercitazioni del corso di Product Image Design (aborotory L'incomprenLibilì sibilita degli ideogrammi viene sopperita dalla presenza del linguaggio non verbale delle icone che presentano identiche in entrambe le versioni del software.

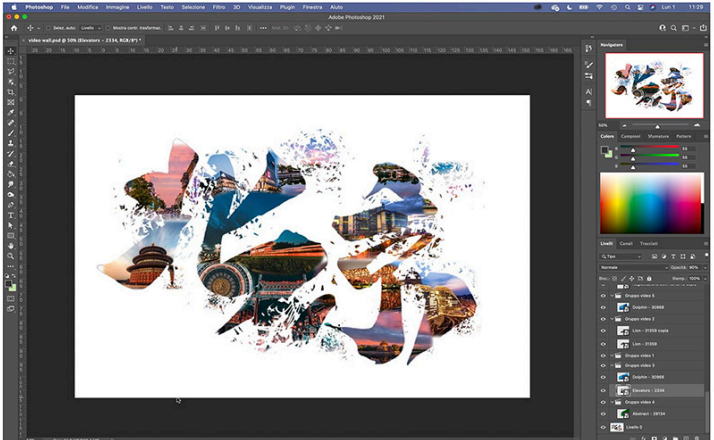

L'esperienza didattica presso la Beijing University of Chemical Tecnology divenuta necessariamente a distanza ha comportato inevitabilmente l'aggiornamento e la rivisitazione dell'approccio rispetto alla modalità in presenza, pur nel mantenimento delle finalità e delle medesime metodologie: piattaforma Teams, tavoletta grafica e organizzazione con alternanza di momenti ex cathedra e momenti di revisione individuale. Un'efficace miscela di soddisfazione e di buoni risultati che hanno visto protagonisti gli studenti cinesi impegnati nell'apprendimento dei principi base della comunicazione grafica computerizzata e nell'approccio critico-conoscitivo degli strumenti e dei metodi necessari per organizzare, elaborare e rappresentare immagini grafiche bitmap e vettoriali attraverso l'utilizzo dei principali software al servizio della rappresentazione e della comunicazione.

Ed è stata proprio l'interfaccia utente di questi software che ha generato in me - e certamente anche negli studenti cinesi che hanno seguito il corso - un certo senso di disorientamento, dovuto prevalentemente alla distanza "percettivo-culturale" che, nel caso specifico, si è manifestata attraverso la diversità dei font - orientali/occidentali - caratterizzanti gli strumenti e le funzioni di comando dei software grafici utilizzati. I caratteri cinesi, infatti, seppur costituiti da ideogrammi esteticamente attraenti che rievocano forme e simboli riconducibili a disegni presenti nella realtà, in un certo senso hanno accentuato in me - e in loro - la percezione di divario tra le due realtà geograficamente e fisicamente molto distanti ma contemporaneamente unite grazie alle irrinunciabili tecnologie informatiche.

Sarebbe stato alquanto improbabile ipotizzare che software nati per la rappresentazione e la comunicazione non permettessero il dialogo tra due realtà idiomatiche così differenti. Problematica efficacemente risolta non solo grazie al fatto che tutte le funzioni di questi software grafici sono supportate da informazioni, sia per il testo occidentale sia per quello in cinese tradizionale e semplificato, ma anche e soprattutto grazie alla presenza di un linguaggio iconico universale dei comandi del tutto identico a quello della versione occidentale. Infatti, nonostante la possibilità di utilizzare le versioni in lingua inglese messa a disposizione dalla software house, un'alta percentuale di studenti ha prediletto la lingua madre per la visualizzazione dell'interfaccia utente sul proprio computer.

Ancora una volta il linguaggio non verbale - in questo caso delle icone presenti nei software - ha permesso di sopperire alle comprensibili e aggiungerei temute problematiche comuni- 
cative e le nuove tecnologie hanno permesso di accorciare le distanze. Superate, quindi, le prime difficoltà linguistiche e la discronia dei fusi, l'esperienza cinese è stata alquanto formativa e madre di innumerevoli riflessioni legate prevalentemente all'elevata internazionalità della didattica. (R.T.).

\section{Riferire (Product Image Design Laboratory)}

Fig. 5. Pagina della Bibbia di Gutemberg, Germania XV secolo,Biblioteca Apostolica Vaticana e parte del Sutra del Diamante, testo a stampa Cina IX secolo, British Museum.
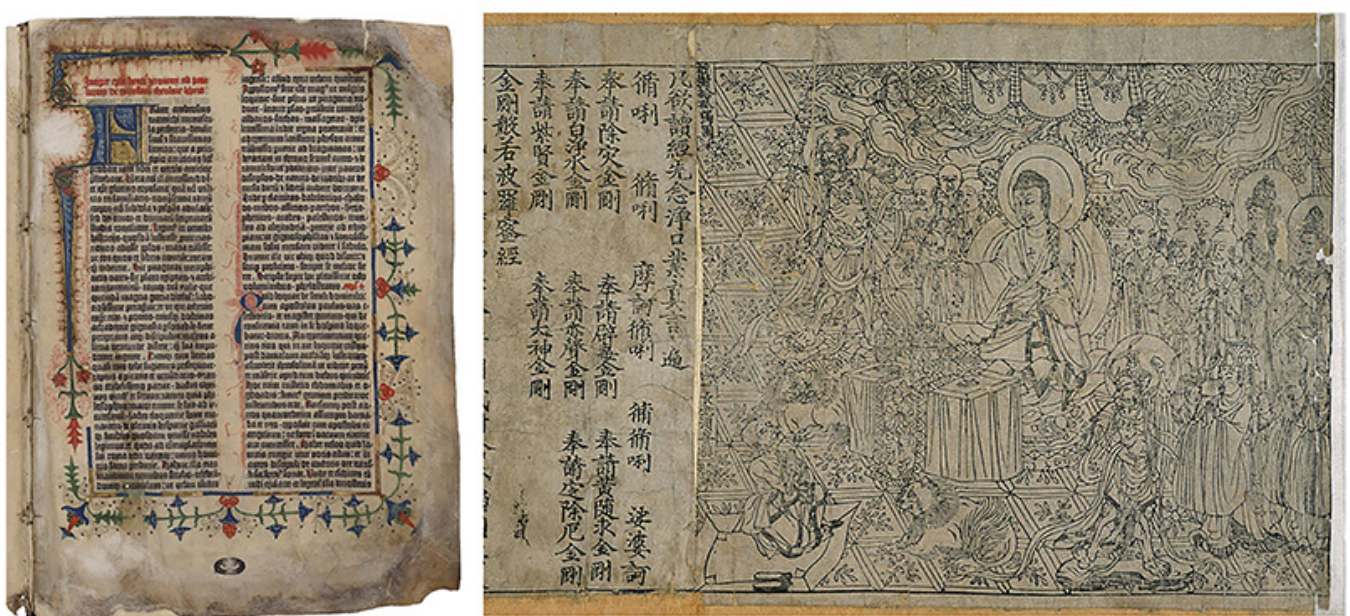

La conoscenza della Storia del Design vede un approfondimento disciplinare nell'ambito specifico della Storia della Grafica, corso che si sviluppa, in particolare, sul tema dell'advertising, inteso come caso studio, a cui riferire l'avvicendarsi di linguaggi e concetti espressi in termini di comunicazione visiva.

Ciò che da subito si è resa evidente, nella necessità di condividere le lezioni di Graphics History con gli studenti della BUCT, è stata la relatività di alcuni punti di vista storiografici che, se possono essere considerati come assodati rivolgendosi a interlocutori con una matrice di studi occidentale, non sono altrettanto scontati per chi proviene da un ambito culturale completamente diverso. La collocazione spaziotemporale, ad esempio, dell'avvento della stampa o del concetto di Rivoluzione Industriale non è univocamente determinata dai due differenti ambiti. Questa considerazione, quindi, ha reso necessario da subito introdurre una serie di puntualizzazioni e precisazioni che hanno caratterizzato lo sviluppo di tutte le lezioni, puntualizzando di volta in volta punti di vista e ragioni a essi correlate.

Ma non solo. II metodo di studio proposto è incentrato, anche per specifica tradizione metodologica del nostro sistema scolastico, sul principio dell'analisi e della comparazione di elementi notevoli all'interno delle varie opere, ma anche in questo caso la conoscenza, se non addirittura la familiarità, con alcuni linguaggi che riportano epoche e stili è divenuta un argomento cruciale per lo svolgimento del corso.

Si tratta cioè di comprendere come una stratificazione di immagini appartenga, come bagaglio culturale, a uno studente occidentale e renda più facile la strutturazione di una lettura più approfondita diacronica: se il ' 700 per noi rappresenta un insieme di suggestioni e riferimenti visivi all'interno dei quali trovare un punto di vista quale quello della grafica pubblicitaria, diversi saranno suggestioni e riferimenti per coloro che hanno un diverso patrimonio visivo all'interno del quale muoversi.

$\mathrm{Da}$ qui quindi la necessità di porre sempre come premessa metodologica riferimenti per immagini e non solo narrativi, per concetti legati a uno specifico riferimento storico, sia esso sociale, architettonico, tecnico o ancora stilistico, poiché II mero riferimento verbale si può trovare, in questo contesto, scevro dal supporto di una mappa concettuale/visiva comune. Il concetto di 'riferimento', quindi, assume un significato più articolato divenendo una specifica proposizione di immagini da cui dedurre concetti e analisi critiche. 
Da qui derivano ulteriori considerazioni che fanno riferimento alla specifica modalità di analisi di una immagine da parte di ambiti culturali così diversi proprio per quanto attiene alla cultura visiva: la scomposizione in differenti piani secondo un concetto legato all'impostazione prospettica, ad esempio, è meno immediata per chi ha nella propria cultura una tendenza a forme compositive in cui il senso di profondità, per tradizione, è meno accentuato. $E$ ancora: la capacità di modulare forma e distribuzione del testo secondo una composizione di volta in volta legata allo stile di un autore appare spesso difficile da analizzare nelle sue sfumature per coloro che non trovano la medesima impostazione compositiva nell'insieme dei propri esempi figurativi a cui fare riferimento.

Da queste riflessioni, quindi, scaturisce la necessità di approfondire una modalità di analisi critica degli esempi proposti, nell'ambito dello sviluppo dell'esperienza didattica a cui si fa riferimento, secondo una logica che non dia per scontata la capacità di individuazione di elementi specifici e che definisca, soprattutto con l'ausilio di immagini, paradigmi di riferimento comuni, utili alla traduzione di concetti sostanziali. (ME.R.).

\section{Note}

[I] A tale proposito, si rimanda all'attività di workshop internazionale Visuality _03 (2020) Appunti istantanei di un'architettura in movimento. Lumen motus movet hominem, con relatori Gabriele Pierluisi (École Nationale Supérieure d'Architecture de Versailles) e Livio Sacchi (Dipartimento di Architettura, Università degli Studi "G. d'Annunzio" Chieti-Pescara); Franco Purini, Carmen Andriani, Manuel Gausa (dAD - UNIGE).

[2] Beijing University of Chemical Technology, Corso di Laurea - con Doppio titolo (Italiano - Cinese) - in Design del Prodotto e della Nautica (UNIGE)/Design del Prodotto (BUCT).

[3] Per una trattazione dell'argomento, si veda: Falcidieno 2006.

[4] Appare opportuno rimandare alla differenza tra 'linguaggio' e 'stile', messa a punto da Gianfranco Caniggia e più volte ripresa negli anni seguenti anche da chi scrive, secondo la quale il linguaggio deriva da un'espressione propria e lo stile dal perseguire modelli sovente anche in maniera passiva.

[5] Fondamentale argomento di ricerca trattato in recenti convenzioni e progetti da Francesca Fatta.

[6] A supporto di quanto detto, basti pensare al cruciale momento di emergenza a tutti i livelli; infatti, è sufficiente ricordare come comportamenti di superamento del limite abbiano anche contribuito a incrementare l'attuale pandemia, fino al disastro causato dagli allevamenti intensivi di visoni, contagiati da Sars-Cov-2, trasmesso e trasmettibile all'uomo <www.fondazioneveronesi.it/magazine/articoli/danonperdere> (consultato il 20 marzo 202I).

\section{Riferimenti bibliografici}

Caniggia G., Maffei G. L. (1979). Lettura dell'edilizia di base.Venezia: Marsilio Editore.

Caniggia G., Maffei G. L. (1994). Composizione architettonica e tipologia edilizia I. Firenze: Alinea.

Faiola A., Macdorman K. F. (2008). The Influence of Holistic and Analytic Cognitive Styles on Online Information Design:Toward a communication theory of cultural cognitive design. In Information, Community \& Society, n. I I, pp. 348-374.

Falcidieno M. L. (2006). Parola disegno segno. Comunicare per immagini. Segno, significato, metodo. Firenze: Alinea.

Falcinelli R. (20|4). Critica portatile al visual design. Torino: Einaudi.

Falcinelli R. (2020). Figure. Torino: Einaudi.

La Penna A. (a cura di). (1967). Orazio - satire ed epistole. Firenze: La Nuova Italia.

Nisbett R. E., Miyamoto Y. (2005). The influence of culture: holistic versus analytic perception. In Trends in Cognitive Sciences, $\mathrm{n}$. 9, issue 10, pp. 467-473.

Pinotti A., Somaini A. (2016). Cultura visuale. Immaggini, sguardi, media, dispositivi. Torino: Einaudi.

Tatler B.W. et al. (2010). Yarbus, eye movements, and vision. In i-Perception, n. I, pp. 7-27.

\section{Autori}

Maria Linda Falcidieno, Università di Genova, marialinda.falcidieno@unige.it

Enrica Bistagnino, Università di Genova, enrica.bistagnino@unige.it

Alessandro Castellano, Università di Genova, alessandro.castellano@unige.it

Massimo Malagugini, Università di Genova, massimo.malagugini@unige.it

Ruggero Torti, Università di Genova, ruggero.torti@unige.it

Maria Elisabetta Ruggiero, Università di Genova, mariaelisabetta.ruggiero@unige.it

Per citare questo capitolo: Falcidieno Maria Linda, Bistagnino Enrica, Castellano Alessandro, Malagugini Massimo, Torti Ruggero, Ruggiero Maria Elisabetta (2021). Modus in rebus/Modus in rebus. In Arena A., Arena M., Mediati D., Raffa P. (a cura di). Connettere. Un disegno per annodare e tessere. Linguaggi Distanze Tecnologie. Atti del $42^{\circ}$ Convegno Internazionale dei Docenti delle Discipline della Rappresentazione/Connecting. Drawing for weaving relationship. Languages Distances Technologies. Proceedings of the $42^{\text {th }}$ International Conference of Representation Disciplines Teachers. Milano: FrancoAngeli, pp. 1615-1632. 


\title{
Modus in Rebus
}

\author{
Maria Linda Falcidieno \\ Enrica Bistagnino \\ Alessandro Castellano \\ Massimo Malagugini \\ Ruggero Torti \\ Maria Elisabetta Ruggiero
}

\section{Abstract}

The focused theme is strictly connected to the recent events linked to the pandemic and to the periods of total enclosure; we have learned to live with ourselves or with a very close family circle and we have learned to use information technology as a "friendly" support and not as a mere program to be learned. We realized the physical distances, which we believed we had canceled forever with the ability to travel in shorter and shorter times. We have experienced the loneliness and the sense of marginality of existence.

But -referring to the teaching and research university experience- all this has also contributed in a surprising way to strengthening our way of working: our cultural and operational role undoubtedly has been enriched, to the point of going beyond the correct balance between relationship "in presence" and "at a distance", up to confusing the plans, as if physical distances were the only impediment to effective communication.

In these brief notes, instead, we want to highlight that there are other "distances", not physical, but perhaps even more limiting for mutual understanding and how a didactic opportunity was the beginning for theoretical-methodological reflections and approach to research on this issue.

Keywords

signs, graphics, culture, belongingness.

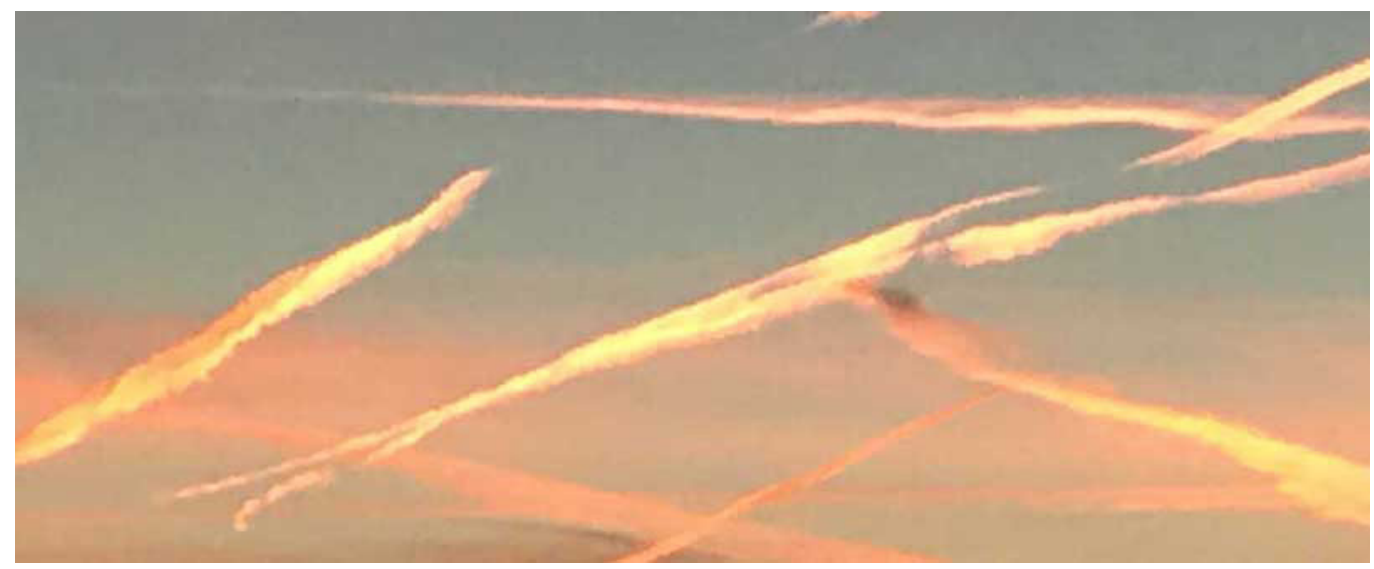




\section{Introduction}

There is a time for everything and there is a modality (of development, perception, appropriation...) for everything. The theme of communicative distance linked to technologies, that allow it to be overcome, and to the possible languages that derive from the use of these technologies appears as an indispensable opportunity to reflect and to understand potential unusual points of view, but certainly useful for a correct understanding of the problem (and / or opportunity) that the current pandemic moment has clearly highlighted [ $\mathrm{I}]$.

Here, however, we want to deal with a peculiar aspect of communication, which sees distance not only as a 'physical' distance, but also as a 'perceptual-cultural' distance, partly resolvable with tools, techniques and methodologies of approach, but partly eliminable (or, at least, resizable) only with a change of approach and with the awareness that the whole subject is in 'sharing' and not only in the speed and power of the contemporary available tools.

The occasion: teaching the disciplines of graphics at a Chinese university [2]; the theme: language (verbal and non-verbal); the perspective of investigation: the relationship that binds new technologies to the possibility of shortening the distance between an eminently visual cultural heritage (that of the expressiveness of oriental peoples) and one divided into alphabetic and image expressions.

Hence, the experience that technology does not always bring closer, but that this always happens when we share some fundamentals necessary for mutual understanding and how, therefore, awareness of the limits (as well as the potential) of contemporary technologies is necessary to support and accompany them.

Because from the experience still in progress it clearly emerged how sharing in depth and not only at a notional level the patrimony of 'basic' expressions, 'codes' is important, as we -researchers- well known in the field of representation - These are the necessary (if not sufficient) conditions for the communication between issuers and receivers to function without the possibility of misunderstandings, ambiguities, errors; especially in the field of training; the possession of a sharing or at least a deep knowledge of the reciprocal expressive codes means avoiding that sort of prevarication that takes place when whoever supplies the information, and guides the visual design expressions, illustrates and tends to make the design purposes uniform, making them adherent with the own cultural background [3]. In the visual project, if the profound reasons for the choices are not known and understood, if a frame of cultural references is not shared, significant difficulties can be encountered in putting texts and images into a system, in their proportioning, in the organization of hierarchies. and spaces, in the choice of the most suitable typefaces for the target audience and for the purpose of communication; in this case, the linguistic understanding is not at all sufficient to guarantee a good yield of the product: texts in English allow to understand the literal meanings of the message, but all the visuality is dangerously balanced between the dictates of those who at that moment transfer skills and those who implement them, trying to adapt their history to an extremely distant and little known one [4].

In this case, technology helps and is necessary for carrying out distance learning, but nothing can do to bridge the other distance, the deeper and more structural one; in a similar way, the tools made with linguistic codes different for matrix (in substance and for a rough definition: pictographic or alphabetic) make it extremely difficult to operate simultaneously -teacher and learner- to the point of sometimes preventing the creation of that empathy and that spontaneity of relationship which is the primary characteristic for the success of a workshop activity.

Wich are the considerations that arose from the reflections made?

First of all, recognizing the importance and necessity of information technologies and the consequent role assumed in training, which no one denies any more; but immediately after recognizing that a unity of purpose is needed, which goes beyond nationality and the single application of knowledge, to make a shift of view unavoidable: it is necessary to work in the search for a sort of 'Esperanto' [5] of visuality, which establishes certain principles that can guarantee the success of visual projects regardless of their geo-cultural location. Working for a globality intended as sharing of codes and consequent comprehension. 
On the other hand, this is what the moment requires in every area and above all in the sharing of respect for the world that hosts us, and which is sending us increasingly clear signs of failure [6]. So, perhaps, it is not just about Modus in rebus, but the ancient warning about respect for limits and the search for balance:"est modus in rebus. sunt certi denique fines, quos ultra citraque nequit consistere rectum" (Orazio, Satire ed epistole) [La Penna 1967, p. I6].

Five research focuses are proposed below: Reflecting, Perceiving, Diversifying, Sharing, Referring. (ML.F.).

\section{Reflecting (Product Image Design Laboratory)}

Fig. I. Product Image Design laboratory, aa. 2020/2021, professo Enrica Bistagnino. A student's representation on the subject of nonconformism. Beijing University of Chemica Technology,

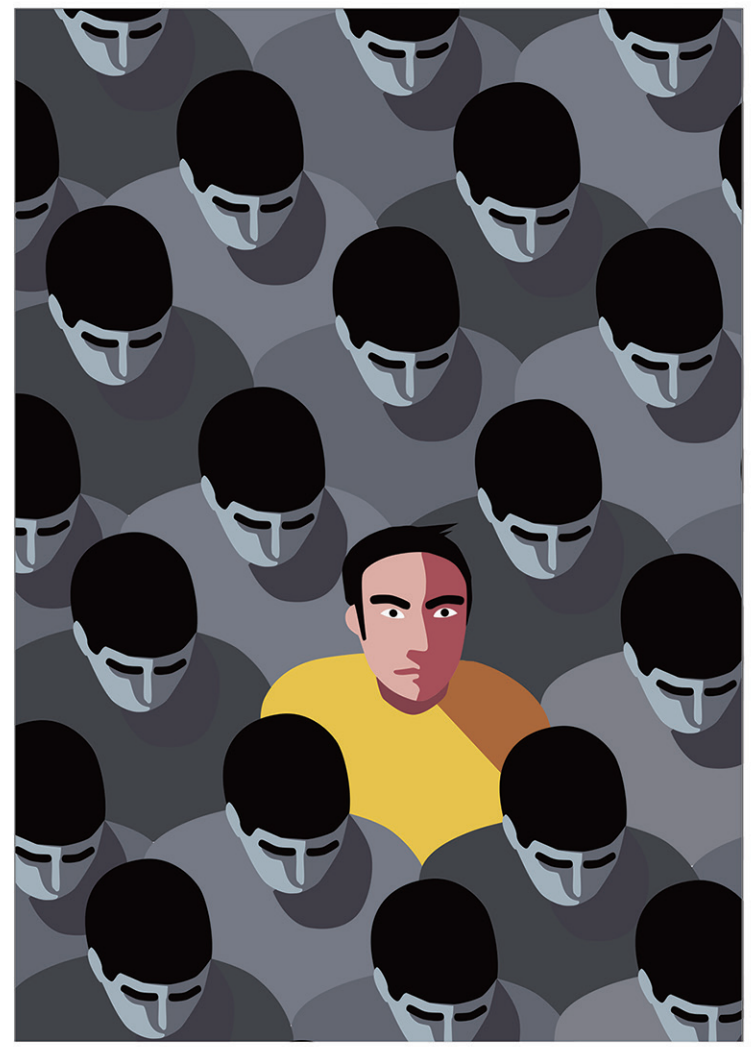

The dialogic power of the image is today more evident and fundamental than ever. Each relationship is mediated by representations of places, people and signs that, mainly in a dynamic form, appear on the multiple interfaces of the web platforms and in the display devices.

As regards the didactic sphere, specifically that relating to the themes of representation and communication, in the experiments carried out an interesting superimposition of images is noted: the object of reflection, a visual artifact, is in fact mediated by further representations which certainly open up many themes for reflection.

In the awareness of the topic complexity, we want, here, only to mention some themes emerged in the didactic experience carried out at BUCT in the Product Image Design laboratory which, on the one hand, has further affirmed the communicative scope of representation, on the other hand it also underlined, as was foreseeable, how important it is, for a deeper knowledge of images 'distant' from our visual language, to access 'in presence' the cultural context that generated them. 
In fact, the exceptional proximity made possible by remote teaching platforms seems to suffer, however, from a general lack of depth and breadth of vision.

In the flatness and delimitation of the screen, the diversity of codes and languages emerging in the images, in some way, results in residual portions of a culture that remains distant, still not fully accessible.

In observing these images that 'open' in the interfaces of the devices, there is no reinforcement generated by the context which constitutes a precious sort of echo of aesthetics and cultures; the landscape is missing, a scenario full of cultural clues; the freedom of the gaze to discreetize signs by selectively orienting one's attention is lacking.

In other words, visuality, delegated to audio-vision, while confirming the 'cosmopolitan' character of visual language, requires that fundamental visual enhancement given by the multisensory activation still strongly linked to processes immersive 'real' rather than virtual connections.

Therefore, if on the one hand the screen of digital devices has allowed, in this period of pandemic, a substantially continuous connection between individuals of the planet from a personal, social and working, educational point of view; if it made it possible to create a sense of community, representing, through people, a sort of 'metaphysical' space, acted virtually, on the other it revealed the limits of the interface in distance learning which, framed on the screen, detects and expresses diversity yet to be fully understood and harmonized in a cultural horizon that cannot be reduced to the mediation of distance communication tools. The students of the BUCT were invited to select some contemporary graphic projects, of oriental culture, and to analyze their structure and codes. The results were interesting, the images decidedly "powerful", but what more strength and clarity they would have had if we had observed and contextualized them in an exchange of glances in a space on campus. (E.B.)

\section{Perceiving (Product Image Design Laboratory)}
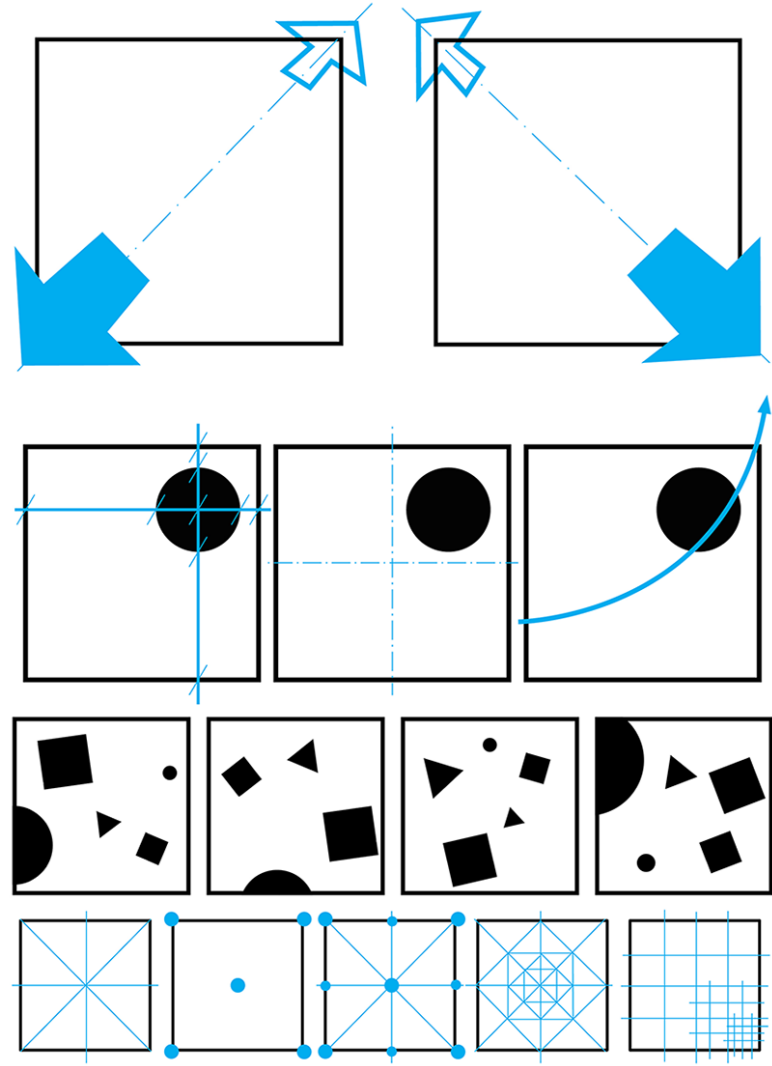
Teaching visual perception lessons in China and at a distance was a challenge right from the start. Perceptual phenomena are usually explained according to some concepts related to the experience of everyday life. For example, to explain the forces active in a field, there is nothing more useful than referring to our reading system: the trend from left to right and that from top to bottom indicate the directions and verses of the two "forces" that cause the bottom right corner of a page to be the heaviest part of the field.

But how to deal with the issue of a writing model so different from ours? In traditional Chinese, writing and reading proceed from top to bottom and from right to left; contemporary Chinese, on the other hand, demonstrates greater flexibility and has adopted left-to-right writing and, on many occasions, also the horizontal trend of writing, probably in order to adapt itself to the language of the world wide web which has imposed a more western organizational model of the page

But if on the one hand the approach of contemporary Chinese to the Western writing model could be seen as a facilitating element on the teaching of perception with the creation of a shared visual context, on the other hand, there remains the doubt of how and how much the rooted model of classical writing can affect the very way of perceiving reality.

During the lessons, the Chinese students demonstrated a particular ability to learn and use the phenomena of perception related to the forces generated by the field and the objects it contains. With some ease they understood the concepts related to harmonic or disharmonic diagonals and to the fatefull rise or fall effect of compositions built on those same elements of the field. But they showed that they understood the concept based on reading images, they could also make the concept to them in the papers created during the course.

With the same ease the students tackled the exercises of visual exploration of the image based on the examples related to the research on eye movements conducted by Alfred L. Yarbus, managing to grasp the necessary details to respond to the different visual tasks. The discussion is different concerning the theme of visual hierarchies. If from a theoretical point of view the concepts were learned without problems, in practice the Chinese students have shown a lower organizational capacity of the elements according to the importance of the individual parts.

What we observed in class gives credence to the theory that binds our way of perceiving and designing, intrinsically linked to the dominant cognitive style with which we learn during our training. The cognitive style is strongly influenced by the socio-cultural context in which we grew up and, several studies, report as "two extremes" the holistic or global model typical of cultures that see the natural order in the relationship between things, as in most oriental cultures, and the analytical model, which has its basis in the Platonic model that sees it in the categorization and analysis of individual objects. Hence the ease of Chinese students to learn relational phenomena, functional and strongly linked to the context and the lesser capacity for discretionary organization of the individual elements. (A.C.).

\section{Diversifying (Product Image Design Laboratory)}

Language is the capacity, peculiar to human beings, to communicate thoughts, express feelings and, in general, to inform other beings about their inner or outer reality, by means of a system of vocal or graphic signs. In an even broader sense, it can be understood as the faculty of expressing oneself through other signs, such as gestures, symbols. The word language can also have a different meaning: it can be understood as the complex of words and locutions that a people uses to express, communicate, exchange thoughts or feelings. The two meanings are inevitably intertwined and, while on the one hand they lead to full understanding between individuals, on the other, they can lead to a 'Babel' of misunderstandings and differences.

This risk can occur when we have to deal with the theme of visual communication -and therefore of the visual code- with subjects coming from countries and cultures particularly 
Fig. 3. After experiments comparing phonetic and ideographic codes in an attempt to visualise the meaning of the word itself they tried to apply the graphes to apply the graphemes to ide alphabet that would be alphabet that would be able to evoke it visually. The term 'al phagram'
was introduced (students was introduced (students
Hong Liu and Chen Boyu).

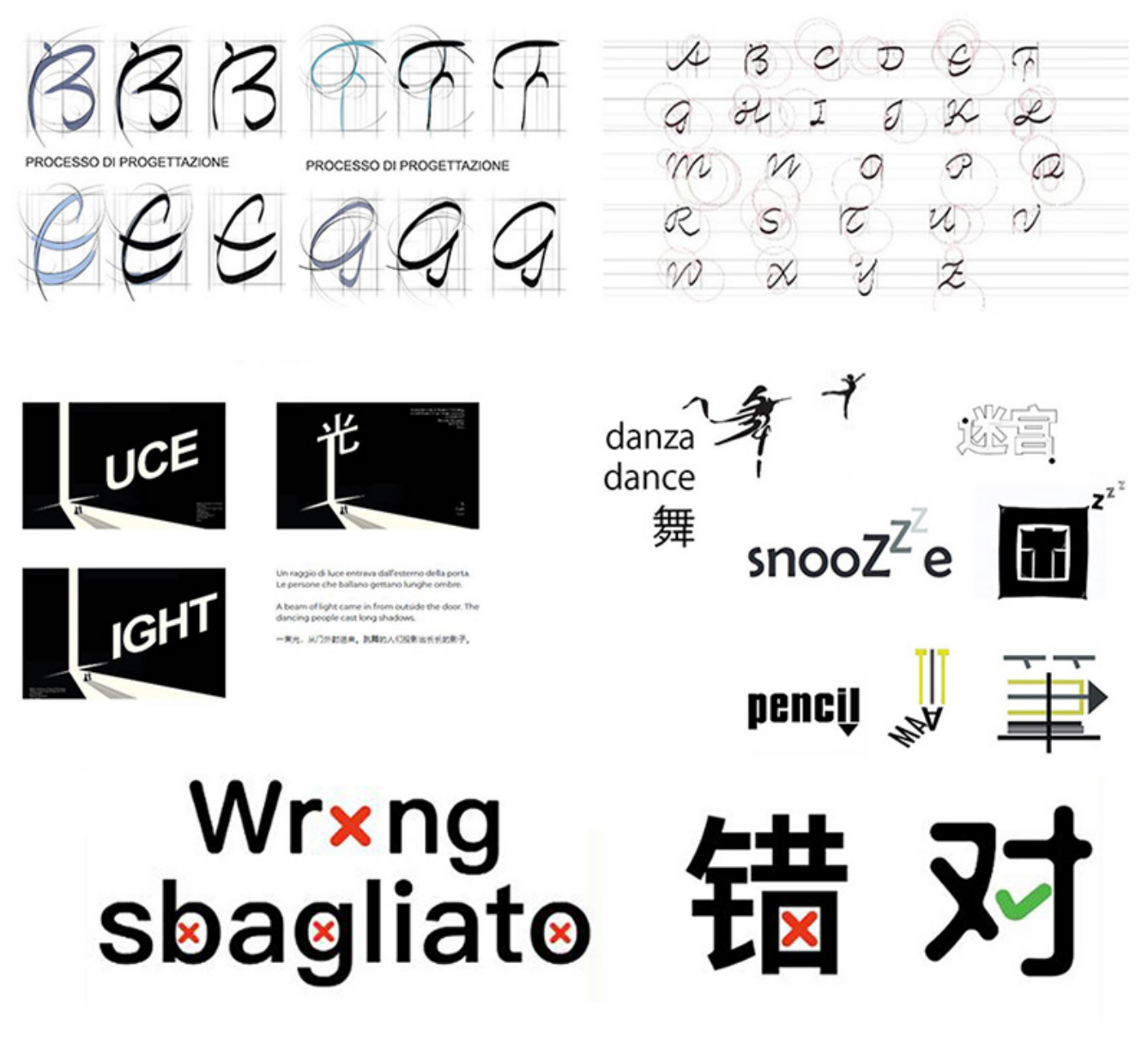

\section{$B B B$ \\ PROCESSO DI PROGETTAZION \\ PROCESSO DI PROGETTAZIONE \\ 999}

o $s \pi \quad \pi /$

N) $x$ is $z$

pencil

distant from our own, as happened in the context of the Product Image Design Laboratory. The great differences, which already emerge from the study of the simplest visual codes linked to colour, become even more evident when we analyse the word, that is, the textual component that is an integral part of communication [Falcidieno 2006], since, in addition to the different meanings that each language attributes to equivalent words (obviously translated), there is a different logic in the graphic representation of the word itself. We have compared a culture that bases its writing on an ideographic system, with ours, which is based on a phonetic system. The problem is not, then, only of a different writing and/or pronunciation of the same term, but of a completely different graphic representation that, inevitably, leads to equally different visual perceptions.

In this context, the analysis of graphic projects that elaborated the textual component so as to highlight the meaning of the word itself from a visual point of view was exemplary. This operation was particularly interesting because it was applied not only to phonetic writing (such as Italian or English), but also to ideographic writing such as Chinese. The work highlighted different approaches to the visual aspects of the written word and showed contrasting sensitivities in taking into account different stylistic meanings in the elaboration of a visual product. If for our culture it is obvious to associate different visual meanings to a different type of font, serial range, upper or lower case, this is not the case in oriental culture. The opposite experiments, i.e. those that try to graphically translate a phonetic alphabet according to graphemes inspired by ideographic writing, have also led to interesting experiments that attempt to find almost impossible connections between such different codes. (M.M.). 


\section{Sharing (Digital Representation and Product Image Design Laboratory)}

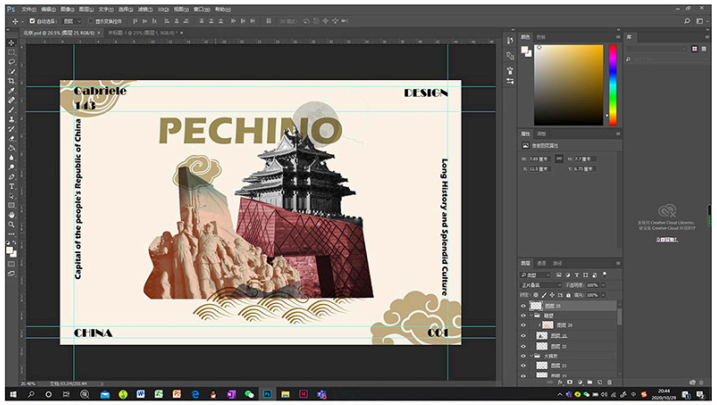

Fig. 4. Comparison between the user interface of the Adobe Photoshop software in Chinese and in Italian used by Chinese students to carry out the exercises of the Product lmage gn Laboratory course The gn Laboratory course. The incomprehensibility of the ideograms is compensated by the presence of the non-verbal language of the icons that are identical in both versions of the software.

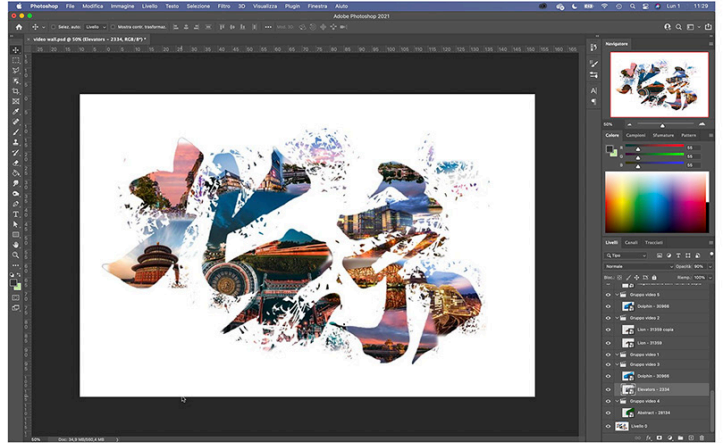

The teaching experience at the Beijing University of Chemical Technology which has necessarily become remote has inevitably entailed updating and revisiting the approach with respect to the face-to-face mode, while maintaining the aims and the same methodologies: Teams platform, graphic tablet and organization with alternation of ex cathedra moments and moments of individual revision. An effective blend of satisfaction and good results that saw Chinese students as protagonists engaged in learning the basic principles of computerized graphic communication and in the critical-cognitive approach of the tools and methods necessary to organize, process and represent bitmap graphic images and vectorial through the use of the main software at the service of representation and communication.

And it was precisely the user interface of these software that generated in me -and certainly also in the Chinese students who took the course- a certain sense of disorientation, mainly due to the 'perceptual-cultural' distance which, in this specific case, it manifested itself through the diversity of fonts -eastern/western- characterizing the tools and control functions of the graphic software used. The Chinese characters, in fact, although made up of aesthetically attractive ideograms that evoke shapes and symbols attributable to drawings present in reality, in a certain sense have accentuated in me -and in them- the perception of the gap between the two geographically and physically very distant realities. but at the same time united thanks to the indispensable information technologies.

It would have been rather unlikely to hypothesize that software born for representation and communication did not allow the dialogue between two so different idiomatic realities. Problem effectively solved not only thanks to the fact that all the functions of these graphic software are supported by information, both for the Western text and for that in traditional and simplified Chinese, but also and above all thanks to the presence of a universal iconic language of the commands of all identical to that of the western version. In fact, despite the possibility of using the English language versions made available by the software house, a high percentage of students preferred their mother tongue for viewing the user interface on their computer.

Once again, the non-verbal language -in this case of the icons present in the software - has made it possible to compensate for the understandable and I would add dreaded 
communication problems and the new technologies have made it possible to shorten the distances. Having overcome, therefore, the first linguistic difficulties and the spindle, the Chinese experience was somewhat formative, and the mother of countless reflections mainly linked to the high internationality of teaching. (R.T.).

\section{Referring (Product Image Design Laboratory)}

Fig. 5. Page of the Gutenberg Bible, Germany I5th century, vatican Apostolic Library and part of the Diamond Sutra, printed text, China 9th century, British Museum.
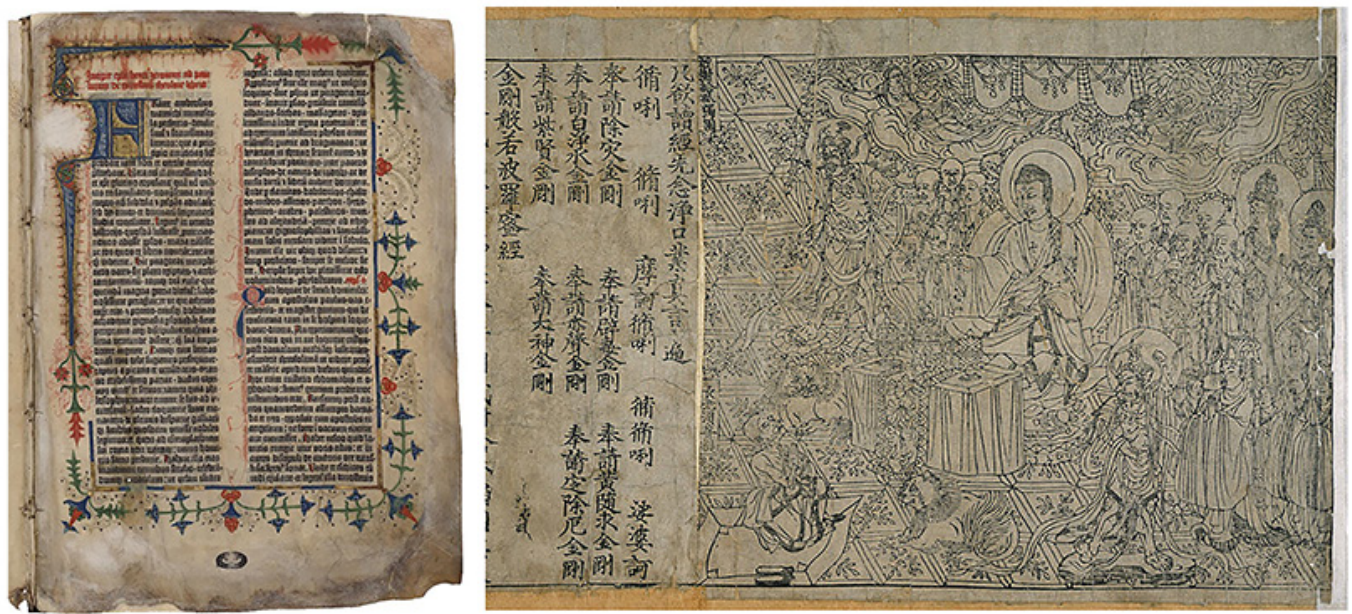

The knowledge of the History of Design sees a disciplinary study in the specific field of the History of Graphics, a course that develops, in particular, on the theme of advertising, understood as a case study, to which the succession of languages and concepts expressed in terms of visual communication refers.

What immediately became evident, in the need to share Graphics History lessons with BUCT students, was the relativity of some historiographical points of view which, if they can be considered as established by addressing interlocutors with a matrix of occidental studies, are not so obvious for those coming from a completely different cultural background. The spatial-temporal location, for example, of the advent of the press or of the concept of the Industrial Revolution is not univocally determined by the two different spheres.

This consideration therefore immediately made necessary the introduction of a series of clarifications and clarifications that characterized the development of all the lessons, pointing out points of view and reasons related to them from time to time.

But not only. The proposed study method focuses, also due to the specific methodological tradition of our school system, on the principle of the analysis and comparison of notable elements within the various works, but also in this case knowledge, if not familiarity, of some languages that refer to epochs and styles it has become a crucial topic for the development of the course.

In other words, it is a question of understanding how a stratification of images belongs, as a cultural background, to a occidental student and makes it easier to structure a more indepth diachronic reading: if the eighteenth century represents for us a set of suggestions and visual references within which to find a point of view such as the one of advertising graphics, there will be different suggestions and references for those who have a different visual heritage.

Hence the need to always place, as a methodological premise, references for images and not just narratives, for concepts linked to a specific historical reference, be it social, architectural, technical or stylistic, since the mere verbal reference can be found, in this context, free from the support of a common conceptual / visual map.

The concept of 'reference' therefore takes on a more articulated meaning, becoming a specific proposition of images from which critical concepts and analyzes can be deduced. 
From these concepts, further considerations derive, referring to the specific method of analysis of an image by such different cultural spheres precisely with regard to visual culture: the division into different planes according to a concept linked to the perspective approach, for example, is less immediate for those who have a tendency in their own culture, to compositional forms in which the sense of depth, by tradition, is less accentuated. And again: the ability to modulate the form and distribution of the text according to a composition each time linked to the style of an author often appears difficult to analyze in its nuances for those who do not find the same compositional setting in the set of their own figurative examples to refer to.

From these reflections therefore the need arises to deepen a method of critical analysis of the examples proposed, in the context of the development of the didactic experience to which reference is made, according to a logic that does not take for granted the ability to identify specific elements and which define, especially with the help of images, common reference paradigms, useful for the translation of substantial concepts. (ME.R.).

\section{Notes}

[I] In this regard, please refer to the international workshop Visuality_03 (2020) Notes of an architecture in motion. Lumen motus movet hominem, with Gabriele Pierluisi (École Nationale Supérieure d'Architecture de Versailles) and Livio Sacchi (Department of Architecture, University "G. d'Annunzio" Chieti-Pescara); Franco Purini, Carmen Andriani, Manuel Gausa (dAD - UNIGE).

[2] Beijing University of Chemical Technology, Degree Course - with Double degree (Italian - Chinese)- in Product and Nautical Design (UNIGE) / Product Design (BUCT).

[3] For a discussion of the topic, see the volume Falcidieno 2006.

[4] It seems appropriate to refer to the difference between 'language' and 'style', developed by Gianfranco Caniggia and repeatedly taken up in the following years also by the author, according to which language derives from its own expression and style from pursuing models often even passively.

[5] Fundamental research topic treated in recent conventions and projects by Francesca Fatta.

[6] To support what has been said, just think of the crucial moment of emergency at all levels; in fact, it is sufficient to recall how overcoming the limit behavior has also contributed to increase the current pandemic, up to the disaster caused by the intensive mink farms, infected by Sars-Cov-2, transmitted and transmissible to humans: <www.fondazioneveronesi.it/magazine/ articoli/danonperdere> (accessed 202I, March 20).

\section{References}

Caniggia G., Maffei G. L. (1979). Lettura dell'edilizia di base.Venezia: Marsilio Editore.

Caniggia G., Maffei G. L. (1994). Composizione architettonica e tipologia edilizia I. Firenze: Alinea.

Faiola A., Macdorman K. F. (2008). The Influence of Holistic and Analytic Cognitive Styles on Online Information Design:Toward a communication theory of cultural cognitive design. In Information, Community \& Society, n. I I, pp. 348-374.

Falcidieno M. L. (2006). Parola disegno segno. Comunicare per immagini. Segno, significato, metodo. Firenze: Alinea.

Falcinelli R. (20|4). Critica portatile al visual design. Torino: Einaudi.

Falcinelli R. (2020). Figure. Torino: Einaudi.

La Penna A. (a cura di). (1967). Orazio - satire ed epistole. Firenze: La Nuova Italia.

Nisbett R. E., Miyamoto Y. (2005). The influence of culture: holistic versus analytic perception. In Trends in Cognitive Sciences, n. 9, issue 10, pp. 467-473.

Pinotti A., Somaini A. (2016). Cultura visuale. Immaggini, sguardi, media, dispositivi. Torino: Einaudi.

Tatler B.W. et al. (20I0). Yarbus, eye movements, and vision. In i-Perception, n. I, pp. 7-27.

\section{Authors}

Maria Linda Falcidieno, Università di Genova, marialinda.falcidieno@unige.it

Enrica Bistagnino, Università di Genova, enrica.bistagnino@unige.it

Alessandro Castellano, Università di Genova, alessandro.castellano@unige.it

Massimo Malagugini, Università di Genova, massimo.malagugini@unige.it

Ruggero Torti, Università di Genova, ruggero.torti@unige.it

Maria Elisabetta Ruggiero, Università di Genova, mariaelisabetta.ruggiero@unige.it

To cite this chapter. Falcidieno Maria Linda, Bistagnino Enrica, Castellano Alessandro, Malagugini Massimo, Torti Ruggero, Ruggiero Maria Elisabetta (2021). Modus in rebus/Modus in rebus. In Arena A., Arena M., Mediati D., Raffa P. (a cura di). Connettere. Un disegno per annodare e tessere. Linguaggi Distanze Tecnologie. Atti del $42^{\circ}$ Convegno Internazionale dei Docenti delle Discipline della Rappresentazione/Connecting. Drawing for weaving relationship. Languages Distances Technologies. Proceedings of the $42^{\text {th }}$ International Conference of Representation Disciplines Teachers. Milano: FrancoAngeli, pp. $1615-1632$ 